\title{
AFM-in-SEM as a Tool for Comprehensive Sample Surface Analysis
}

\author{
Veronika Novotna, ${ }^{1}$ Josef Horak, ${ }^{1 *}$ Martin Konecny, ${ }^{2}$ Veronika Hegrova, ${ }^{1}$ \\ Ondrej Novotny, ${ }^{1}$ Zdenek Novacek, ${ }^{1}$ and Jan Neuman ${ }^{1}$ \\ ${ }^{1}$ NenoVision s.r.o., Purkynova 649/127, 61200, Brno, Czech Republic \\ ${ }^{2}$ CEITEC BUT, Technicka 2896/2, 61600, Brno, Czech Republic \\ *josef.horak@nenovision.com
}

\begin{abstract}
Key features and applications of a unique atomic force microscope (AFM), the LiteScope ${ }^{\mathrm{TM}}$, which can be integrated into a scanning electron microscope (SEM) is reported. Using the AFM-inSEM as one tool combines the capabilities of both systems in a very efficient way. The LiteScope design features advanced Correlative Probe and Electron Microscopy $(\mathrm{CPEM})^{\mathrm{TM}}$ imaging technology that allows simultaneous acquisition of multiple AFM and SEM signals and their precise in-time correlation into a 3D CPEM view. AFM-in-SEM advantages are presented using several examples of applications and AFM measurement modes including CPEM, material electrical and mechanical properties together with nanoindentation, and focused ion beam (FIB) applications.
\end{abstract}

Keywords: atomic force microscopy, scanning electron microscopy, correlative microscopy, AFM-in-SEM, nanoindentation

\section{Introduction}

A current trend in a surface investigation can be defined, both in academia and industry, as follows: (i) the need to characterize and analyze structures in the (sub)nanometer range is growing; (ii) the complexity of such measurements across various instruments creates a demand for advanced correlative analysis; and (iii) easy-to-use instrumentation and automated procedures are of great interest.

Atomic force microscopy (AFM) and scanning electron microscopy (SEM) are two of the most used techniques for sample nanoscale analysis. To tackle market demands, NenoVision has developed a unique AFM, the LiteScope ${ }^{\mathrm{TM}}$, designed for integration into the SEM. The motivation behind the design is twofold: first, such a hybrid system allows for efficient, yet complex in situ sample characterization, and, second, it brings new possibilities for advanced correlative imaging, which allows sample analysis in a way that was difficult or impossible by separate SEM and AFM systems.

In SEM, the sample surface is scanned with a focused primary electron beam [1]. This interaction produces various signals that, through a range of detectors, provide different information about the surface and its chemical composition [2]. In combination with focused ion beam (FIB), SEM is also used for surface modification. In general, a 2D SEM image provides great material contrast and a large depth of focus [3], all in a wide range of imaging resolutions down to around $1 \mathrm{~nm}$.

On the other hand, an AFM scans the sample surface with a sharp tip and measures attractive and repulsive forces emerging between the tip and the sample [4]. Thanks to this principle, AFM can provide sub-nanometer resolution combined with information on topography, roughness, mechanical, and magnetic and electrical properties depending on the probe used. Thus, the integration of AFM inside a SEM combines the capabilities of both techniques and brings many advantages.
AFM-in-SEM integration enables true correlative imaging where the region of interest (ROI) on the sample is analyzed by AFM and SEM at the same time. Traditionally, the correlation is achieved by imaging the sample by a number of techniques, subsequently followed by data analysis and final image overlay, for example, correlative Raman imaging and scanning electron microscopy (RISE) is in this category [5]. There are also approaches for simultaneous measurements of multiple signals such as correlative light-electron microscopy (CLEM) that combines an optical microscope with an SEM into one system [6]. Common issues with traditional approaches are either high initial investment into sophisticated, dedicated instruments or problematic image correlation due to data incompatibility (data formats, pixel count and size, etc.) between devices used [7]. LiteScope is equipped with Correlative Probe and Electron Microscopy (CPEM) ${ }^{\mathrm{TM}}$ technology that enables simultaneous data acquisition and correlation of multiple channels; for example, the material contrast given by SEM is merged with the 3D topography of surface features obtained by AFM, resulting in a 3D CPEM view (Figure 1).

Another key advantage of the AFM-in-SEM approach is in situ measurement, meaning minimal sample handling and contamination due to vacuum preservation inside the SEM, which is crucial for sensitive samples. Also, using SEM, the AFM tip can be navigated with nanometer precision to the ROI, which makes obtaining measurements extremely timeefficient and eliminates the need for navigation marks made by FIB milling.

Lastly, the entire system is highly customizable (for example, scanner configuration, immersion lens compatibility) and can be equipped with many additional accessories, such as a nanoindenter or tweezers, that further broadens possibilities of the AFM-in-SEM concept for complex and efficient sample analysis.

This article demonstrates the abilities and advantages of CPEM technology in AFM-in-SEM applications as well as use of the LiteScope as a stand-alone AFM. Technical aspects of the system, including self-sensing probes and supported measurement modes, are detailed in the Methods section. The applications discussed cover a combination of key features and measurement modes including topography in combination with material, electrical, and mechanical properties and FIB milling applications.

\section{Materials and Methods}

LiteScope is a compact AFM microscope $(129 \times 90 \times$ $45 \mathrm{~mm}$ ), designed to fit into the SEM chambers of most microscope manufacturers (TESCAN, Zeiss, ThermoFisher Scientific, 

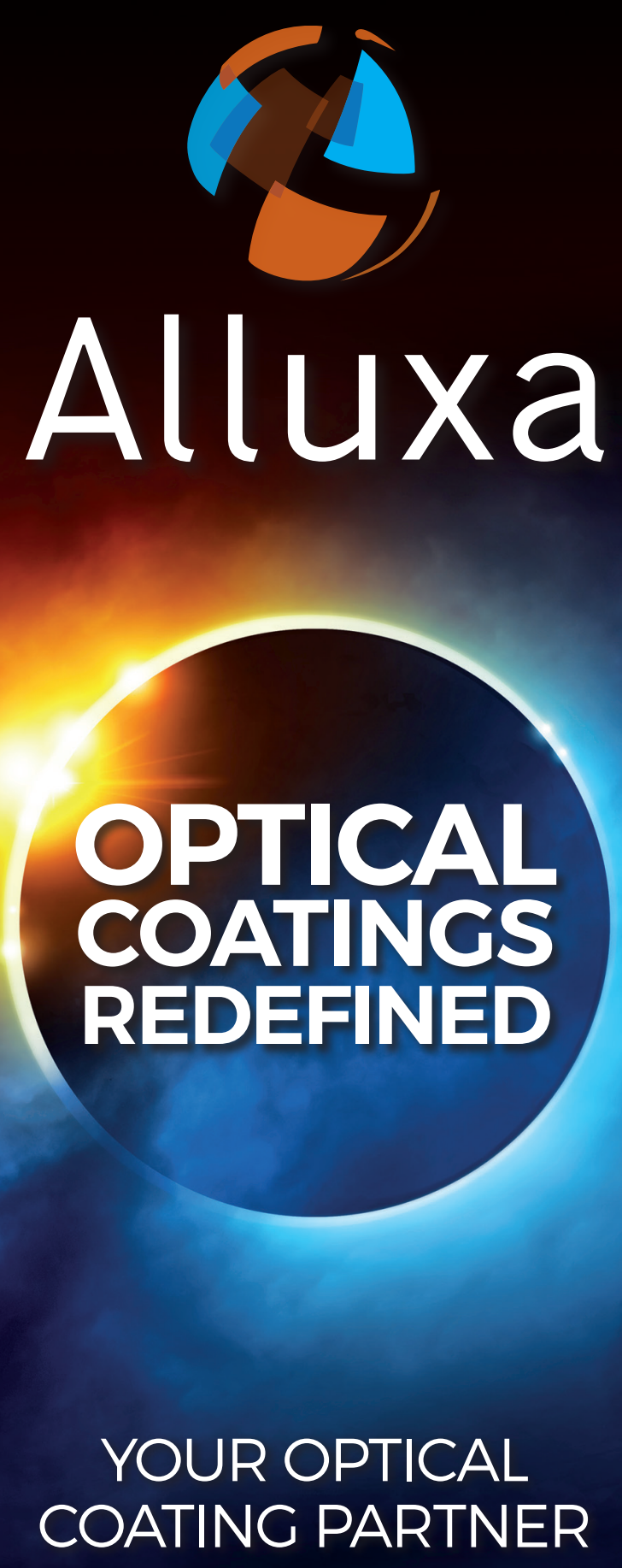

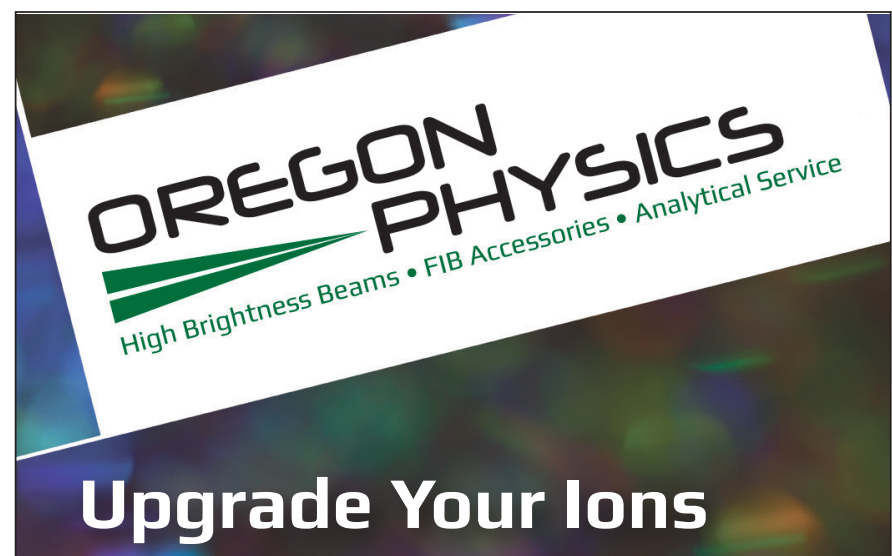

Hyperion ${ }^{\text {TM }}$ Dual Polarity Ion Sources are now available as direct upgrades from Oregon Physics for FEI FIB 200, PHI Adept 1010, and Cameca NanoSIMS, IMS F series, and 12XX series instruments.

Upgrade your ion source to benefit from:

- Longer source lifetime

- Better image resolution

- Improved depth profiling (SIMS)

- Higher currents for milling (FIB)

Oregon Physics' Hyperion ion sources are designed to bolt-on to your existing optical system for easy implementation.

How will Hyperion improve your research? Learn more at Oregon-Physics.com or call us to discuss your requirements.

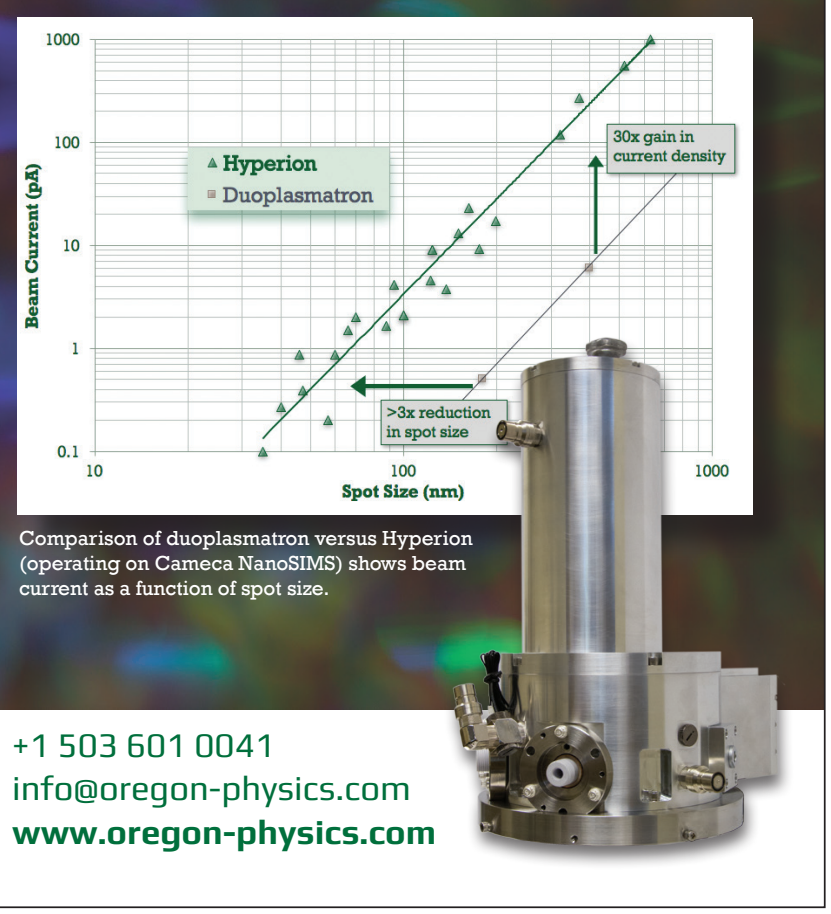


(a)

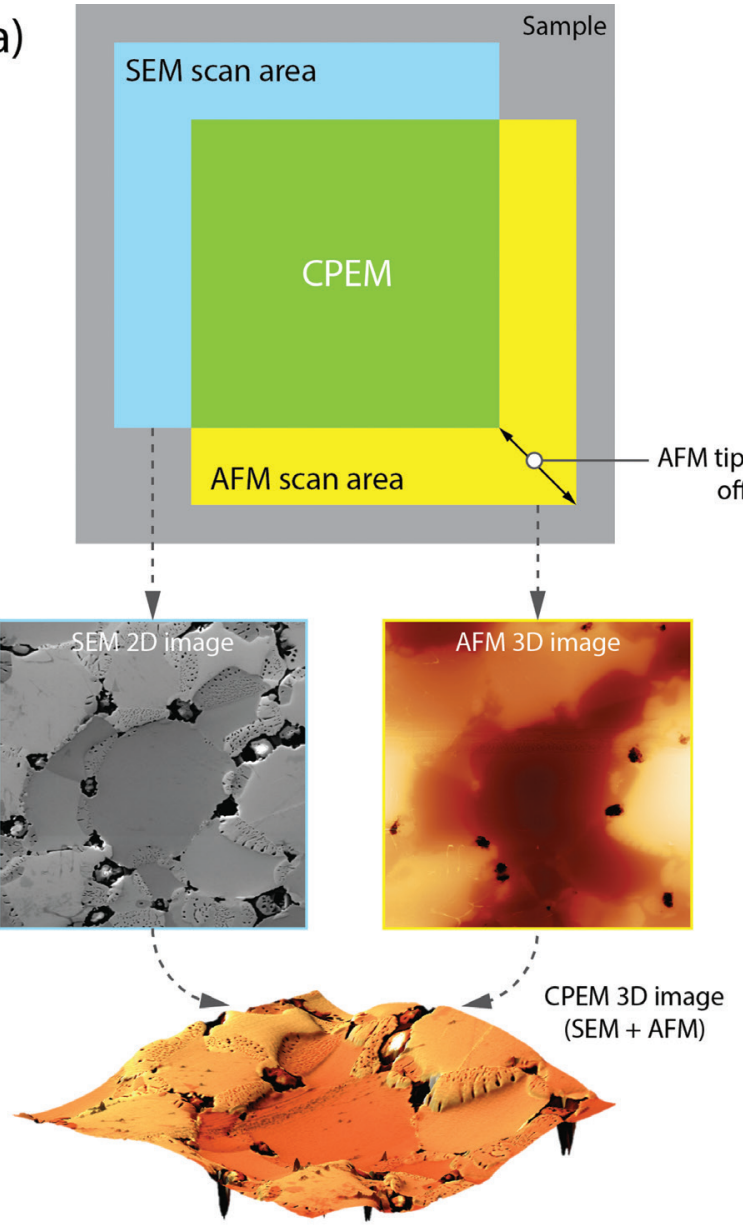

(b)

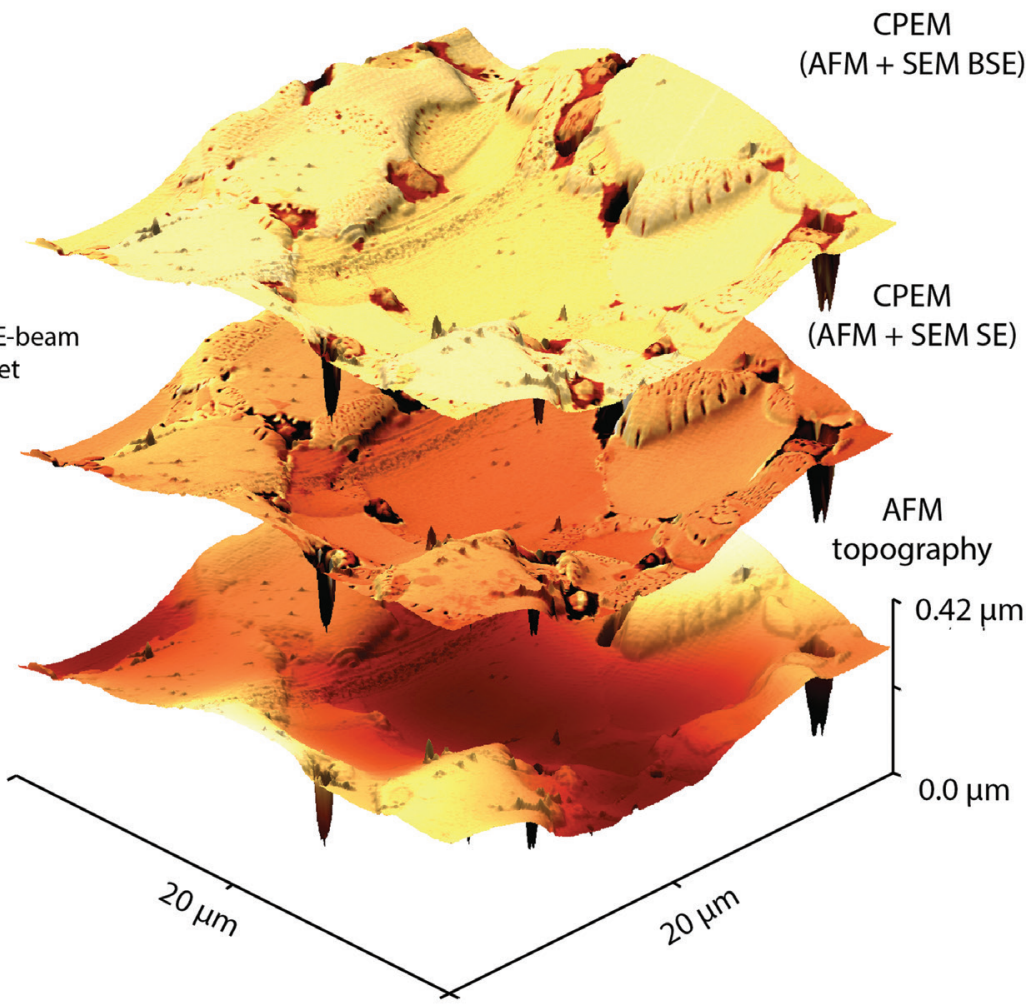

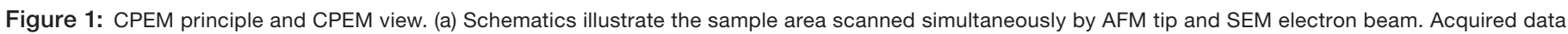

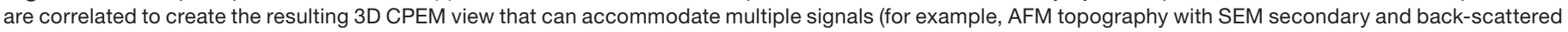
electrons), as demonstrated by a tungsten-chromium sample containing hafnium oxide particles (b).

Hitachi, JEOL) and can be easily mounted on the SEM stage through a dedicated adaptor (Figure 2). It is a highly customizable plug-and-play solution with optional scanner configurations (both in open- and closed-loop mode) with a version compatible with magnetic immersion SEM, and installation takes only a few minutes. The standard configuration offers a large scanning area up to $100 \times 100 \times 100 \mu \mathrm{m}$ and a resolution down to $0.2 \mathrm{~nm}$. An optional scanner configuration improves resolution to $0.07 \mathrm{~nm}$ and decreases the scanning area to $38 \times 38 \times 38 \mu \mathrm{m}$. Further customization includes specimen exchange through the SEM load-lock system [8] or addition of a nanoindenter module or specialized sample and probe holders.

The system includes the NenoBox ${ }^{\mathrm{TM}}$ control unit, which is connected to both the AFM and SEM detectors. This allows simultaneous acquisition and correlation of AFM and SEM signals through CPEM technology. Data are visualized and processed in NenoView ${ }^{\mathrm{TM}}$, a web-based software package that does not require pre-installation or a dedicated computer. Data can be accessed from any device connected to the internet or local network. CPEM technology allows for true data correlation, and some applications are detailed in the Results and Discussion section.
Compatibility with a variety of self-sensing and self-actuating probes operated up to $75 \mathrm{kHz}$, based on a tuning fork principle or piezoresistive cantilever, significantly extends capabilities of the instrument [9]. A list of supported techniques is presented in Table 1. Additionally, self-sensing probes eliminate several disadvantages of traditional AFM cantilevers with optical detection, such as the need for frequent laser alignment, imaging artifacts, and optical diffraction limitations [10]. Use as a dedicated AFM with NenoCase ${ }^{\mathrm{TM}}$, which is designed to dampen vibrations and to allow measurements under different atmospheres, is also possible.

\section{Results and Discussion}

Correlative microscopy. CPEM represents a unique approach to correlative imaging since it enables simultaneous acquisition of SEM and AFM data and precisely merges them. Thus, the differences between material (SEM) and topography (AFM) contrast can be quickly and precisely distinguished. The working principle of CPEM is as follows: the SEM view enables precise positioning of the AFM probe to the ROI on the sample mounted on the LiteScope's piezo scanner. Then, the electron beam is focused near the AFM tip, and the SEM is set to point or spectroscopy mode. The AFM tip, as well as 
(a)

Probe holder with Self-sensing probe a probe inserted
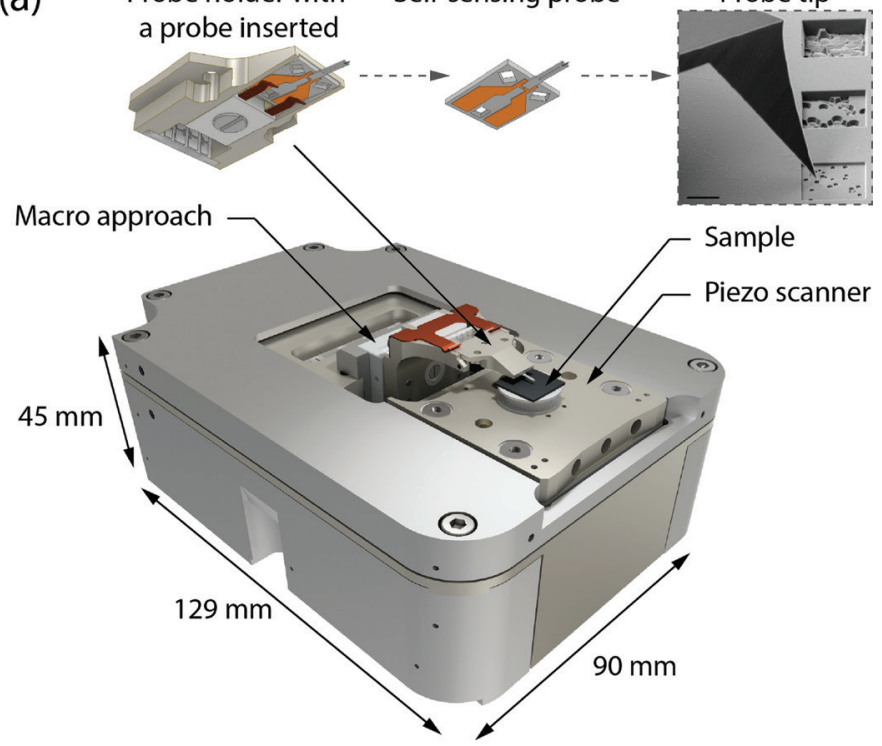

(b)

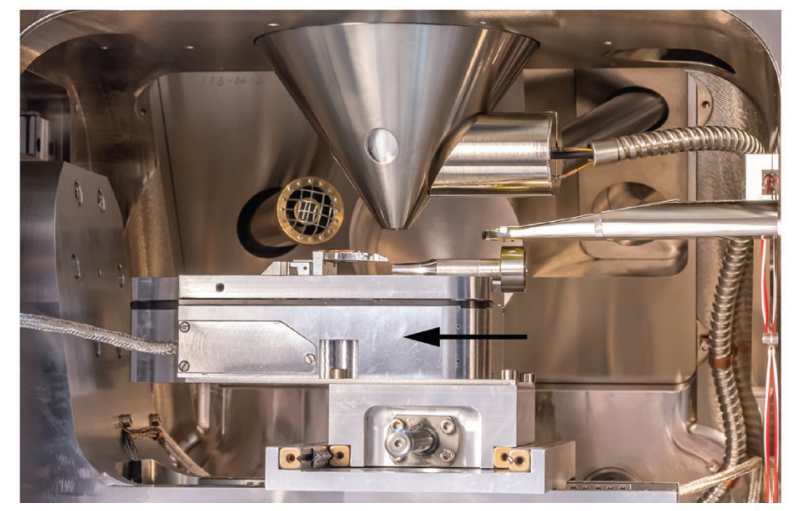

Figure 2: The AFM module can be easily integrated into existing SEMs. (a) Schematics showing the main dimensions and parts of the microscope, including the AFM probe. (b) The arrow highlights the scanhead mounted inside the SEM chamber. the electron beam, remain stationary during data acquisition, and scanning is conducted solely by the piezo scanner. This configuration ensures a constant offset between the AFM and SEM images, which is removed in a postprocessing step [11]. The result is a perfect correlation of multiple signals providing insight into the sample surface properties. The data can be also displayed in a scalable 3D view, which may help facilitate data interpretation (Figure 1a). CPEM can accommodate several types of signals including secondary (SE) and back-scattered electrons (BSE) (Figure 1b), electron beam induced current (EBIC), and cathodoluminescence (CL).

Figure 3 illustrates the use of CPEM on two samples, exfoliated $\mathrm{WSe}_{2}$ flakes on Si nanopillars and a duplex steel sample. Figure 3 a shows a multilayered $\mathrm{WSe}_{2}$ flake, which is deformed by placement on $100 \mathrm{~nm}$ high Si nanopillars. A monolayer of $\mathrm{WSe}_{2}$ is barely visible in the SEM image, but it is clearly visible by AFM topography. Localization of such a small structure by separate AFM and SEM instruments would be an extremely tedious process with a high level of uncertainty, which is eliminated by the AFM-in-SEM approach. The scalable 3D CPEM view of correlated data helps to visualize and understand how the flakes cover the nanopillars. A certain shape of the $\mathrm{WSe}_{2}$ monolayer over the nanopillars creates a single-photon emitter useful for quantum cryptography for secure communications.

Figure 3 b shows a sample of ferritic-austenitic duplex steel. This material exhibits improved material and thermal properties in comparison with either completely ferritic or austenitic steel. This results in higher strength with high ductility, outstanding corrosion-resistance properties, higher impact value, and better conductivity [12]. Nevertheless, failure in duplex steel processing can modify the balance of alloying elements, which leads to a drastic deterioration of its advantageous properties [13].

Features of duplex steel are difficult to identify by a single technique. SEM signals visualize the difference in crystallographic orientation of the twin grains within the same austenite phase and surrounding ferrite matrix. However, the duplex

Table 1: List of measurement modes supported by the LiteScope.

\begin{tabular}{|l|l|}
\hline Mechanical Properties & Application \\
\hline Atomic force microscopy (AFM) & Topography \\
\hline Energy dissipation & Local elastic properties (tapping mode) \\
\hline Force modulation microscopy (FMM) & Local elastic properties (contact mode) \\
\hline Force-distance measurement & Local sample hardness (non-topographic) \\
\hline Nanoindentation & Depth-dependent material characterization \\
\hline Nanomanipulation & Various in situ operations, sample handling \\
\hline Electrical Properties & Application \\
\hline Scanning tunneling microscopy (STM) & Sub-nanometer topography \\
\hline Kelvin probe force microscopy (KPFM) & Local surface potential \\
\hline Conductive AFM (C-AFM) & Conductivity map \\
\hline Conductive CPEM (C-CPEM) & Conductivity map including insulated areas \\
\hline Spectroscopy regimes & Local electrical properties (non-topographic) \\
\hline Electro-mechanical Properties & Application \\
\hline Piezoresponse force microscopy (PFM) & Piezoelectric domain imaging \\
\hline
\end{tabular}


(a)
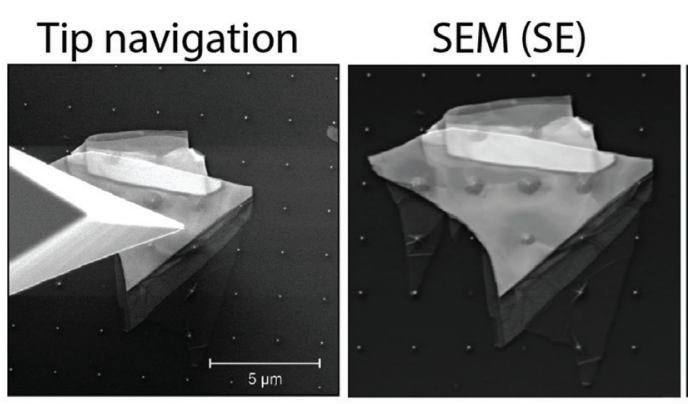

AFM topography
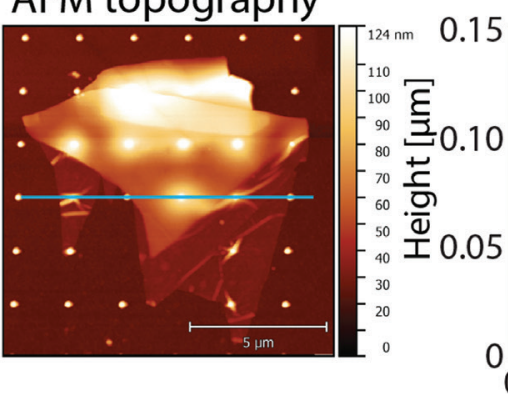

AFM height profile

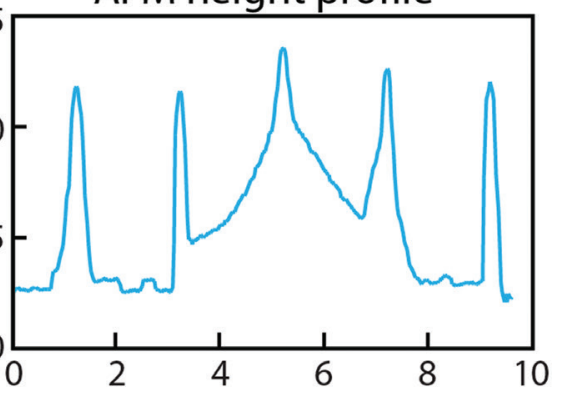

CPEM

Distance $[\mu \mathrm{m}]$

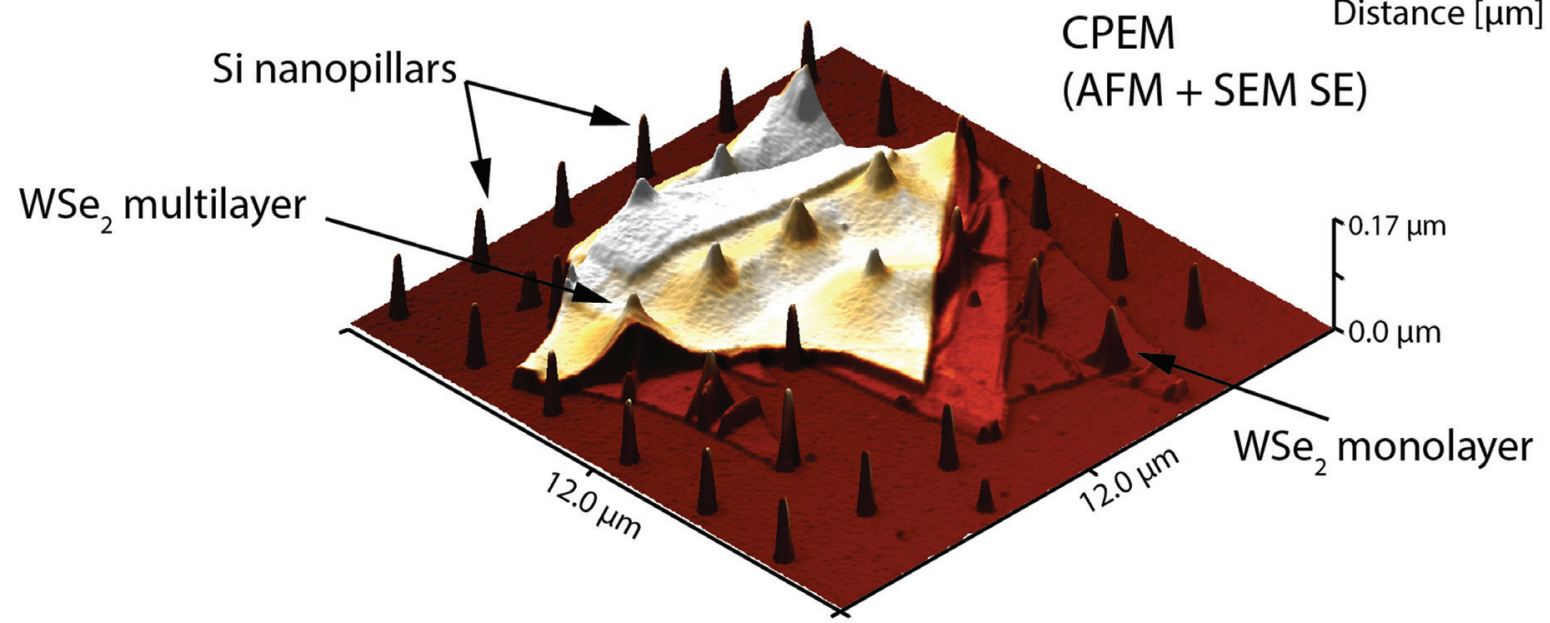

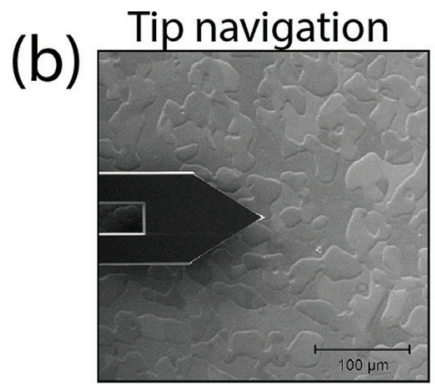

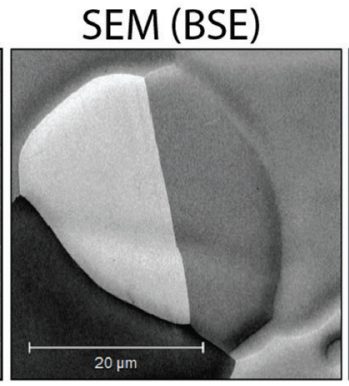

AFM topography

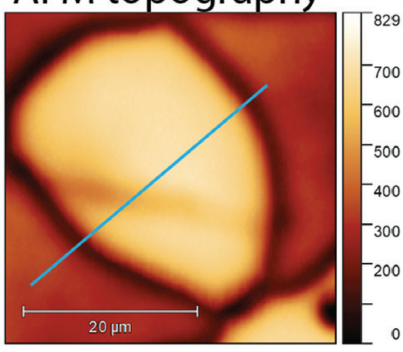

Austenite phase in different crystallographic orientations

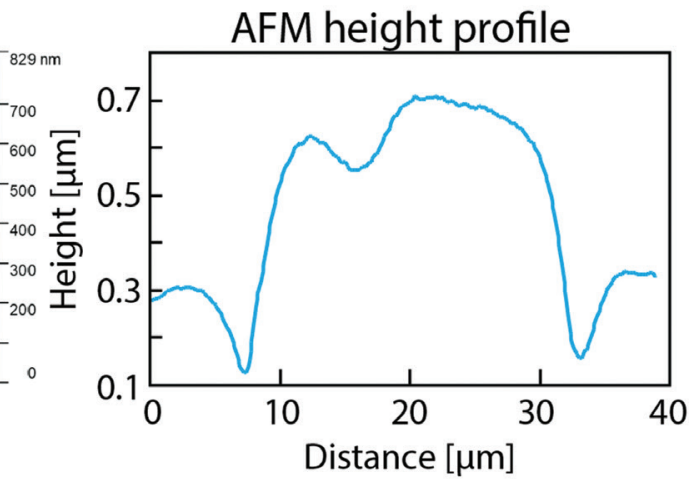

AFM height profile

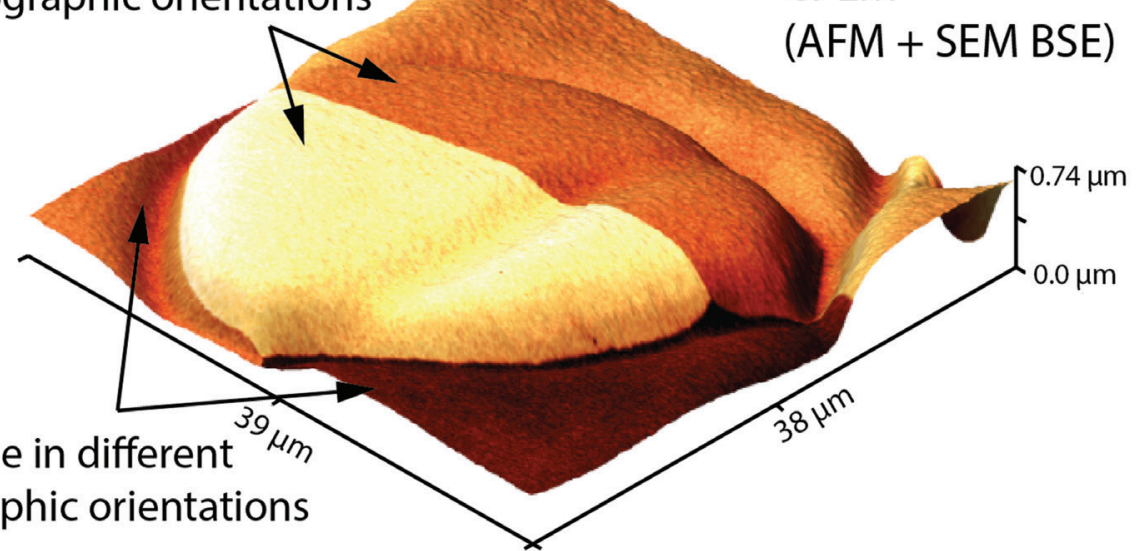

Ferrite phase in different crystallographic orientations

\section{CPEM}

\section{$(\mathrm{AFM}+\mathrm{SEM} B S E)$}

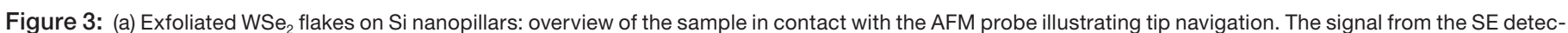

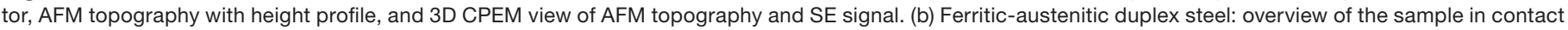
with the AFM probe. The signal from the BSE detector, AFM topography with height profile, and 3D CPEM view of AFM topography and BSE signal. 
(a)

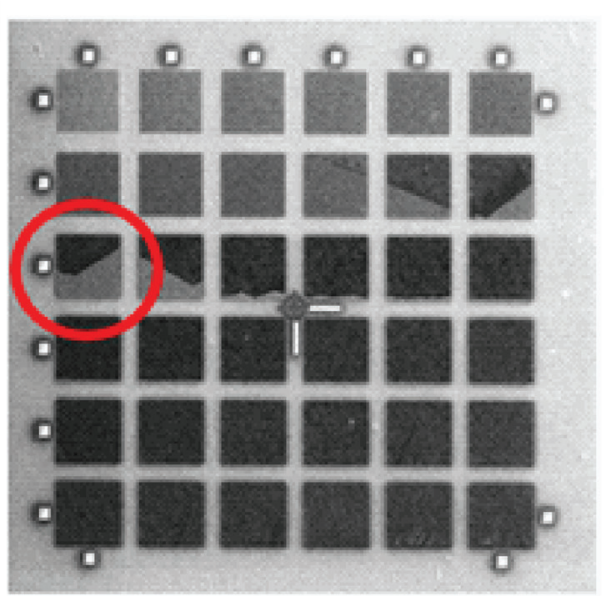

(b)
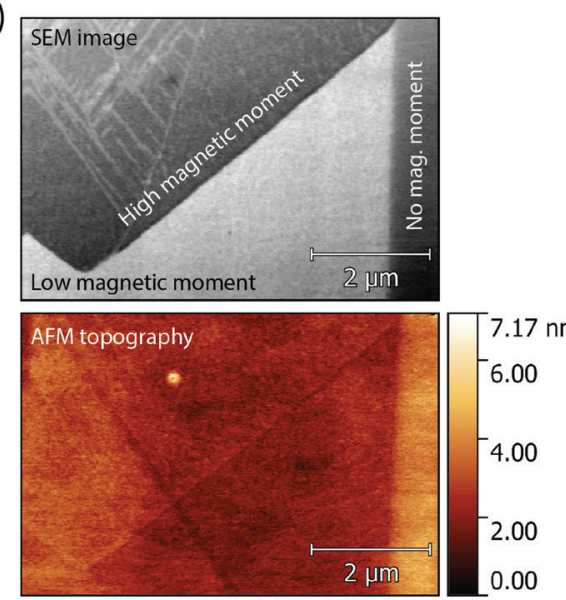

(c)
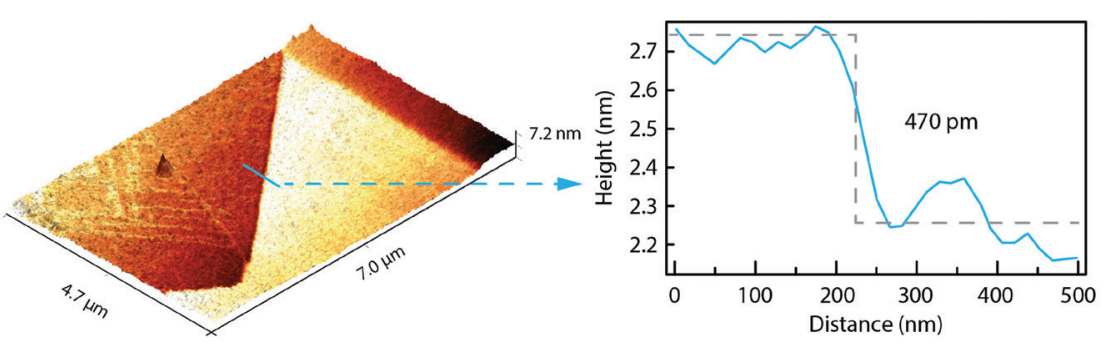

Figure 4: Metastable $\mathrm{Fe}_{78} \mathrm{Ni}_{22}$ thin film on $\mathrm{Cu}(100)$ substrate. (a) An array of FIB-milled squares $(5 \times 5 \mu \mathrm{m}$ each) prepared using different ion dose intensities. The highlighted area shows the partial material crystallography transformation detailed in the SEM image and corresponding AFM topography (b), and combined 3D CPEM view (c) with a topographical step between the high and low magnetic moment.

steel alloy consists of two phases that are only partially dependent on morphology. The SEM images show only the edges of the grains, but information about grain topography is missing. Simultaneously acquired AFM images provide data of the true surface topography. The scalable 3D CPEM view combines crystallographic contrast and surface morphology, enabling easier interpretation of the surface with high resolution in all three dimensions. Information about topography enables precise measurement of the height and depth of surface structures as well as estimation of the sample roughness.

\section{In situ Sub-Nanometer Profiling and 3D Topography}

AFM-in-SEM technology facilitates precise measurement of height and depth of surface structures in SEM applications. It can be operated in a tilted position up to $60^{\circ}$, which makes it compatible with a FIB and gas injection system. This technique is used for surface polishing, sputtering, etching, depositing, or patterning. The results can be immediately viewed by SEM, however, the detailed information about the surface morphology of sputtered or deposited material requires AFM analysis, typically ex situ. The AFM integrated into the FIB-SEM system provides a fast and accurate in situ analysis of the modified surface without the need for sample transfer to another instrument and without changing the environment and breaking the vacuum. Thus, the AFM-in-SEM system in combination with CPEM facilitates FIB process optimization.

Applications for FIB surface modification are depicted in Figure 4, which shows a metastable $\mathrm{Fe}_{78} \mathrm{Ni}_{22}$ thin film grown on

\section{Measurement of Mechanical Properties}

Mechanical properties of a material describe its behavior under loading, and LiteScope offers a variety of methods for measurement of mechanical properties (Table 1). The most studied mechanical properties are stiffness, elasticity, and hardness [15]. The knowledge of these properties is crucial for design, modeling, and analysis of mechanical systems as well as for applications in life sciences. The analysis of mechanical properties on a nanometer scale by multiple techniques provides information about the texture, crystallography, morphology, and properties of individual components and helps to understand the behavior of these systems in the macroscopic world.

A two-component polymer sample, containing polystyrene (PS) and low-density polyethylene (LDPE), was used to demonstrate some capabilities for estimation of mechanical properties; for this purpose, LiteScope is showcased as a stand-alone AFM. Figure 5a shows the topography of the surface with circular regions embedded into a matrix, which can be well correlated with the sample mechanical properties acquired by energy dissipation, FMM, and force-distance curves (Figure 5c). Energy dissipation and FMM provides qualitative information, and both techniques clearly showed a difference in stiffness between the harder PS matrix and the softer, embedded LDPE regions. In general, energy dissipation information is read from the drive signal amplitude in tapping mode and is useful as an alternative to FMM, which uses contact mode AFM. Thus, the application is suitable for samples 
(a) Energy dissipation

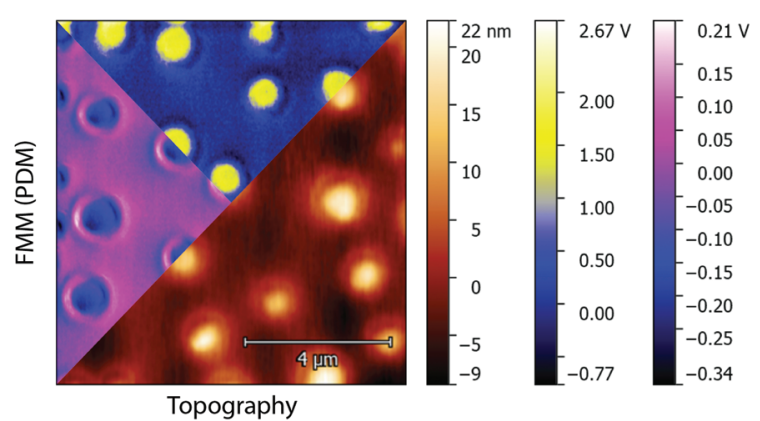

(b)

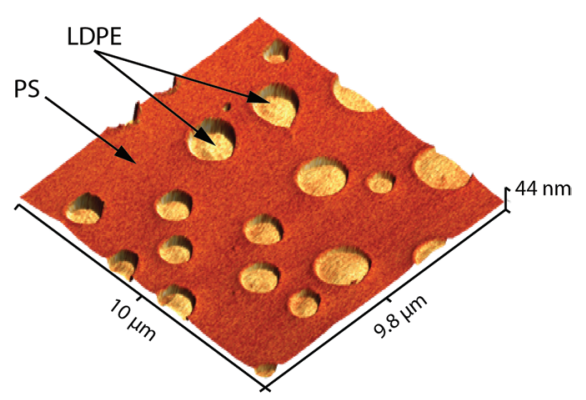

(c)

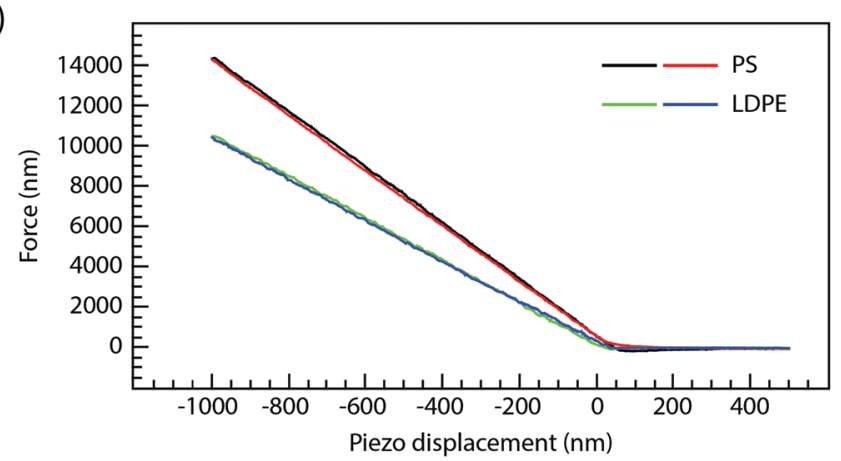

Figure 5: Mechanical properties of the two-component PS and LDPE sample. (a) Sample region showing topography, energy dissipation, and FMM (PDM) image. (b) 3D correlative view showing topography combined with PDM. (c) Difference in slope of force-distance curves demonstrates differences in relative hardness of the PS and LDPE components.

(a)
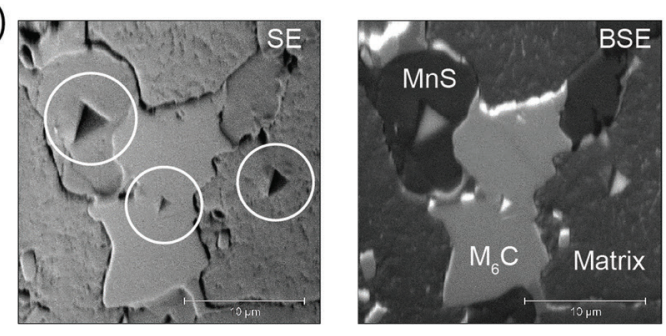

(b)

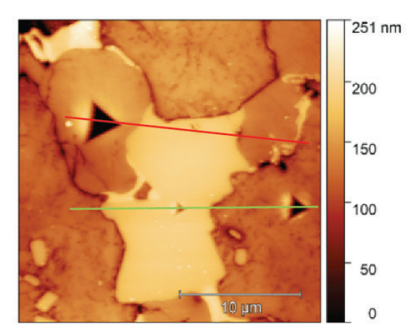

(c)
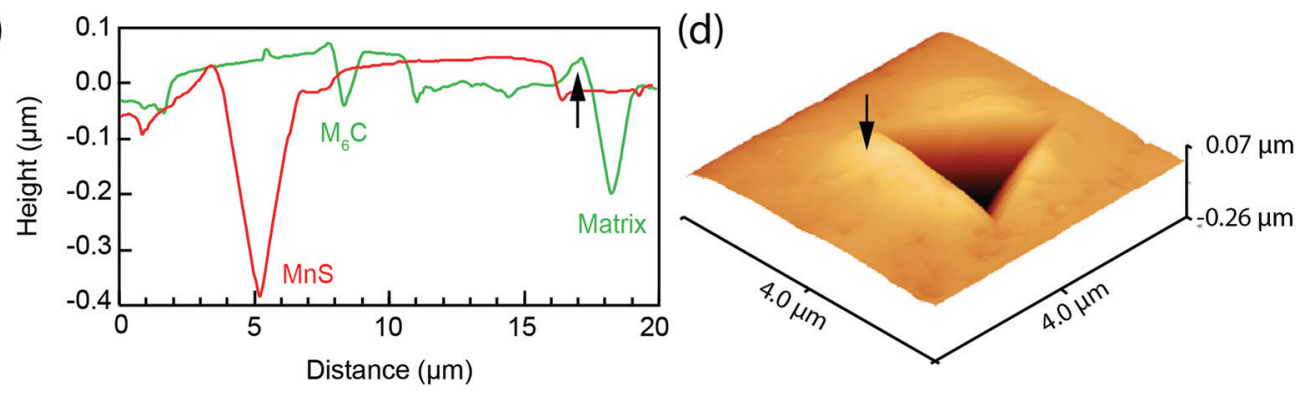

Figure 6: Mechanical properties of an M3 class 2 high speed steel. (a) SE image with circles highlighting individual indents and BSE image indicating the material composition. (b) AFM topography image with profile lines detailed in the depth profile (c) and (d) 3D AFM view of indentation in the matrix. The arrows in (c) and (d) highlight the same area.

FMM image. Acquired data can be viewed in $3 \mathrm{D}$, where the topography together with the mechanical properties improves insight into the surface structure (Figure 5b).

On the other hand, force-distance curves (or $\mathrm{F} / \mathrm{Z}$ spectroscopy) provide a quantitative estimation of the local mechanical property by measuring the vertical force between the AFM tip and the surface, and the sample displacement. It is used for many purposes including sample stiffness analysis, detailed surface-tip force progress, and local elasticity/plasticity determination. The curves (and the slopes) in Figure $5 c$ show a clear difference between the components of the polymer blend with the established material hardness of $2 \mathrm{GPa}$ for PS and 0.3 GPa for LDPE.

The advantages of combining related techniques into one tool are further demonstrated on the nanoindentation of M3 class 2 high-speed steel, which is used in the automotive industry [16]. In general, nanoindentation represents an extremely versatile technique to determine material mechanical properties at the nanoscale such as elastic modulus, hardness values, fracture toughness, or creep and yield stress. In this case, the AFMin-SEM approach was further extended by adding a nanoindenter [17] to the scan head. This hybrid technique enables sample phase identification, precise indentation targeting, and topographical analysis all in one measurement.

Figure 6 shows the etched steel nanoindentation analysis results. The SEM images (Figure 6a) showed a wide range

whose topography is best measured using non-contact mode. In FMM, the AFM tip is scanned in contact with the sample, and an additional driving signal is applied to vibrate the tip, which is converted to a signal used for analysis. Either change in amplitude (data not shown) or phase shift (called phase detection microscopy $[\mathrm{PDM}]$ ) is used to generate the of separate phases of small size $\left(\mathrm{MnS}\right.$ grain, carbide $\mathrm{M}_{6} \mathrm{C}$, and martensitic matrix), that were nanoindented using a force of $15 \mathrm{mN}$. The AFM scans in Figure $6 \mathrm{~b}$ revealed that each phase reacted differently to the acting force. The softest material is the $\mathrm{MnS}$ grain, followed by a matrix, and the hardest is the $\mathrm{M}_{6} \mathrm{C}$ part of the composite, as depicted in the height profile in Figure $6 \mathrm{c}$. 

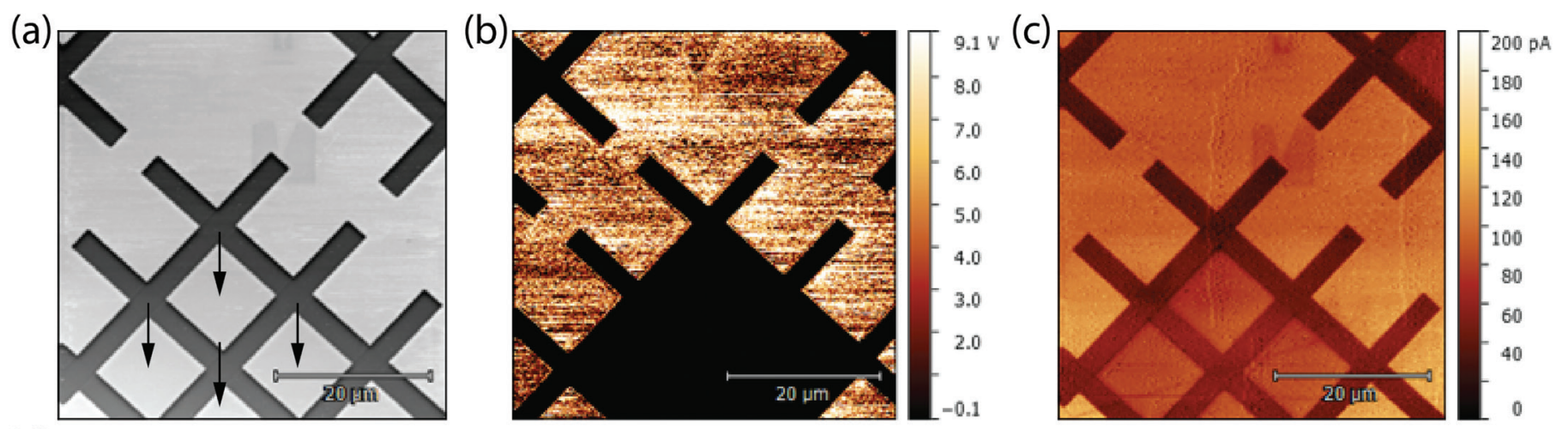

(d)

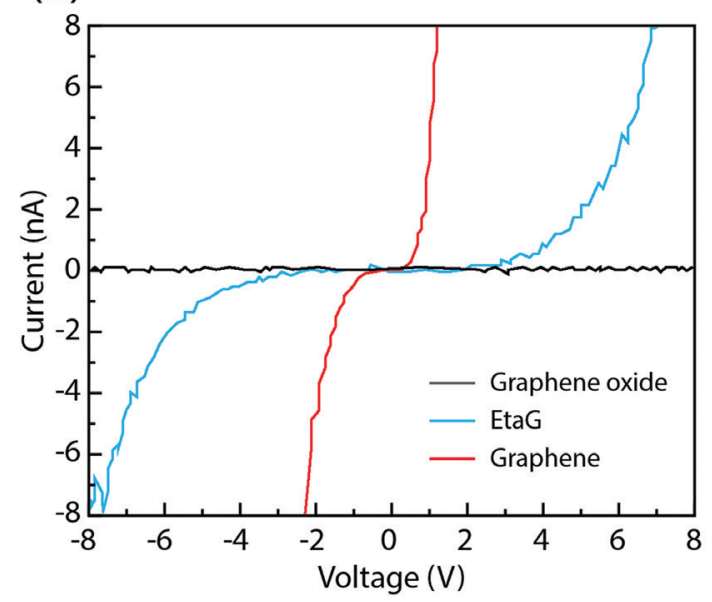

Graphene oxide

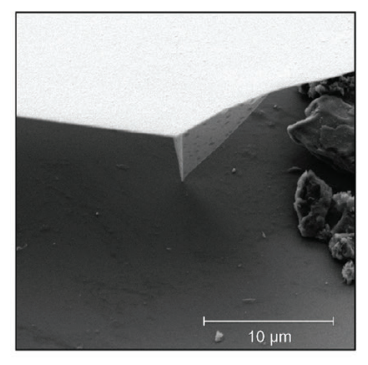

Graphene

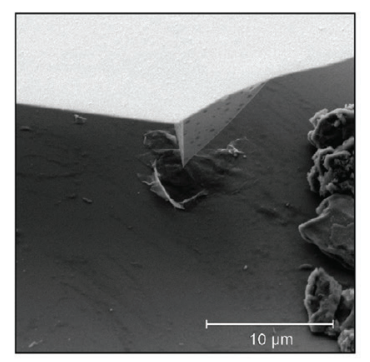

EtaG

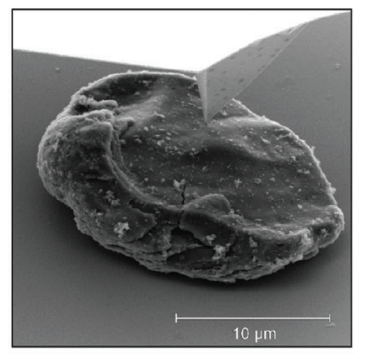

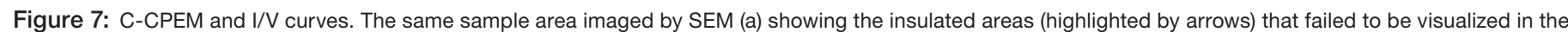

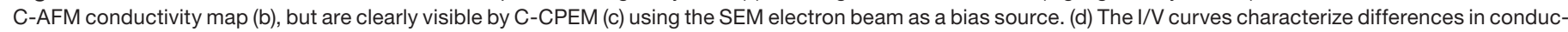

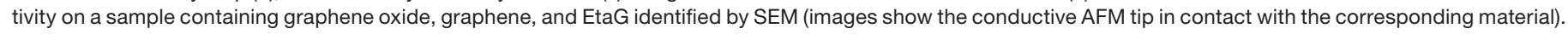

The detailed 3D AFM view shows indentation in the matrix with the amount of material pushed out of the indent (the black arrows in Figures $6 \mathrm{c}$ and $6 \mathrm{~d}$ ).

\section{Measurement of Electrical Properties}

The measurement of electrical properties can also be carried out. This category includes conductive AFM (C-AFM), conductive CPEM (C-CPEM), Kelvin probe force microscopy (KPFM), probe force microscopy PFM, and spectroscopy regimes (Table 1). Technical advancement, especially in the semiconductor and solar industries, places increasing demand on the measurement of current distribution (conductivity) over the sample surface with nanometer precision. Conductive AFM represents a common technique where a bias voltage is applied between the conductive cantilever and the sample, and the resulting current (as well as topography) is measured as the tip scans the sample surface. Clearly, this approach cannot work in insulated areas (Figure 7a), as depicted in Figure 7b. However, this C-AFM limitation can be overcome by the AFM-in-SEM approach combined with C-CPEM technology (Figure 7c), where the electron beam at the constant distance from the tip replaces the need for an applied bias in the measured area, and the tip-sample bias can still be simultaneously applied. During scanning, the tip-sample current flow is measured in contact AFM mode. This means that during a single measurement, information about the sample topography in contact mode, SE and BSE images, and a conductivity map can be acquired.

For a detailed local investigation of the surface electrical properties, AFM-in-SEM can be used to measure currentvoltage characteristics (I/V curves) by applying a bias of $-10 \mathrm{~V}$ to $10 \mathrm{~V}$. Figure $7 \mathrm{~d}$ demonstrates the use of I/V curves for conductivity measurement of a sample containing graphene oxide, graphene, and 4-ethynylaniline graphene derivative (EtaG). The SEM enables precise identification and navigation of the conductive tip to the respective material. The investigation revealed that graphene oxide is non-conductive, EtaG more conductive, and graphene the most conductive material [18].

\section{Conclusion}

This article provides insight into the AFM-in-SEM technology. The LiteScope is a compact, yet powerful, AFM that fits into a large variety of SEM systems in a plug-and-play fashion. It provides complex sample surface analysis by supporting multiple techniques measuring structural, electrical, magnetic, and mechanical properties. The unique CPEM technology allows a number of AFM and SEM signals to be simultaneously measured and automatically correlated into one detailed 3D image while maintaining the same pixel size and coordinate system. The described technology enables complex sample analysis that was either impossible or extremely tedious/expensive when measured by separate instruments. 
Using the SEM to navigate the AFM tip with nanometer precision provides quick localization and study of nanostructures and nanodevices of interest, making analysis precise, comprehensive, and time- and cost-efficient. Combined with FIB, AFM-in-SEM technology offers a range of measurements that are impossible to do with separate stand-alone devices or by other techniques. Additionally, this opens up new possibilities for advanced measurements in the field of metrology, FIB milling optimization, and electron beam induced current or spectroscopy analysis where the combination of AFM and SEM is essential. However, these applications are beyond the scope of this article.

Taken together, the main advantages of the LiteScope are a compact design compatible with most SEM systems, simultaneous in situ measurement of multiple signals, addition of $3 \mathrm{D}$ information to 2D SEM images, a variety of self-sensing and self-actuating probes without laser detection, fast and precise navigation of the probes to the ROI by SEM, a plethora of measurement techniques for comprehensive sample analysis, and web-based software.

\section{Acknowledgments}

This article was created with state support from the Technology Agency of the Czech Republic within the Program for the Support of Applied Research ZÉTA for the project Development of SPM applications suitable for correlative microscopy, project number TJ01000434. The authors also thank all researchers who prepared and provided samples for the analysis.

\section{References}

[1] L Crouzier et al., Nanotechnol 10 (2019) 1523-36.

[2] S Flegler et al., Scanning and Transmission Electron Microscopy: An Introduction, W.H. Freeman and Company, 1997, 65-68.

[3] J Beauvais et al., Proc of SPIE 10291 (1997) 175-99.

[4] N Jalili and K Laxminarayana, Mechatronics 14 (2004) 907-45.

[5] A Matheson, Spectroscopy 33 (2018) 26-28.

[6] P DeBoer et al., Nature Methods 12 (2015) 503-13.

[7] RL Mitchell et al., Microsc Microanal 25 (Suppl 2) (2019) 416-17.

[8] U Kohl-Roscher, Zeiss (2015), https://zeiss.com.

[9] Z Wu et al., Sensors 15 (2015) 28764-71.

[10] M Dukic et al., Sci Rep 5 (2015) 16393.

[11] J Neuman et al., Microsc Microanal 25 (Suppl 2) (2019) 430-31.

[12] M Knyazeva and M Pohl, Metallogr Microstruct Anal 2(2013) 113-21.

[13] A Rocha et al., Stainless Steels and Alloys, ed. Z Duriagina, IntechOpen, London, 2018, 1-14.

[14] M Urbánek et al., Appl Materials 6 (2018) 060701.

[15] S Sundararajan et al., Ultramicroscopy 91 (2002) 111-18.

[16] N Randall et al., Thin Solid Films v. 290-291 (1996) 348-54.

[17] Alemnis, Pushing the Frontier of Nanomechanical Testing, https://alemnis.com.

[18] D Chronopoulos et al., Chem Comm 55 (2019) 1088-91.

\section{Get Radical With Evactron Plasma Cleaning}

\section{Evactron ${ }^{\circledR}$ E50 and E-TC Turbo-Plasma ${ }^{\mathrm{TM}}$ cleaner for your SEM/FIB}

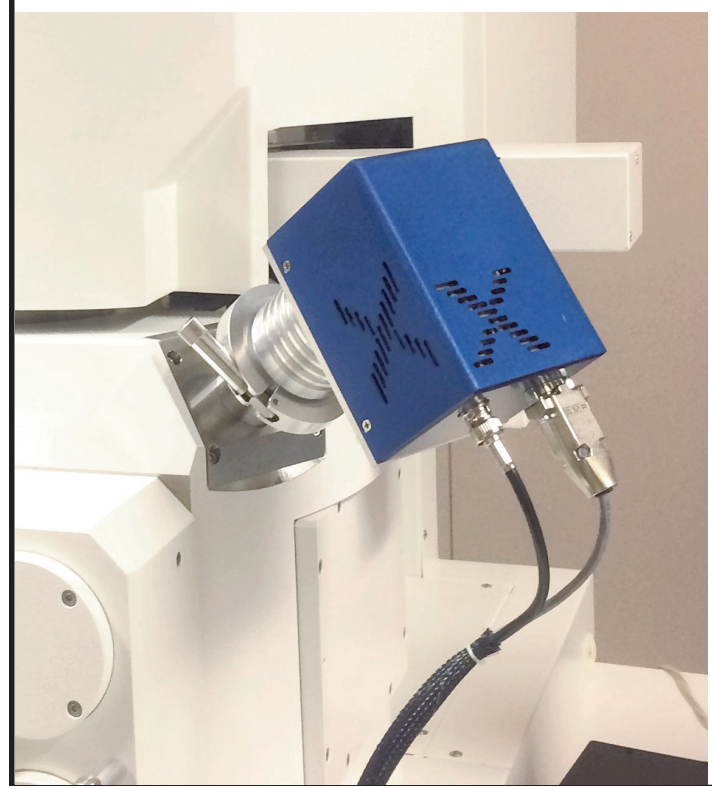

Evactron plasma cleaners generate neutral oxygen radicals from room air. The radicals do the hydrocarbon removal, not the plasma. Features include:

- External hollow cathode plasma radical source

- "POP'т" Ignition at high vacuum-no venting

- Touchpad or Android tablet operation

- Set cleaning time, cycles and power levels

- Cleans in minutes for days of perfect imaging and analysis

WWW.EVACTRON.COM 1-650-369-0133

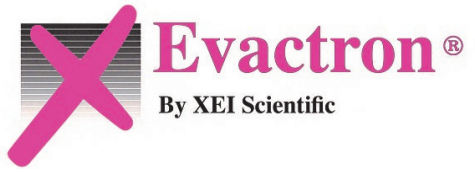




\section{Kammrath Weiss}

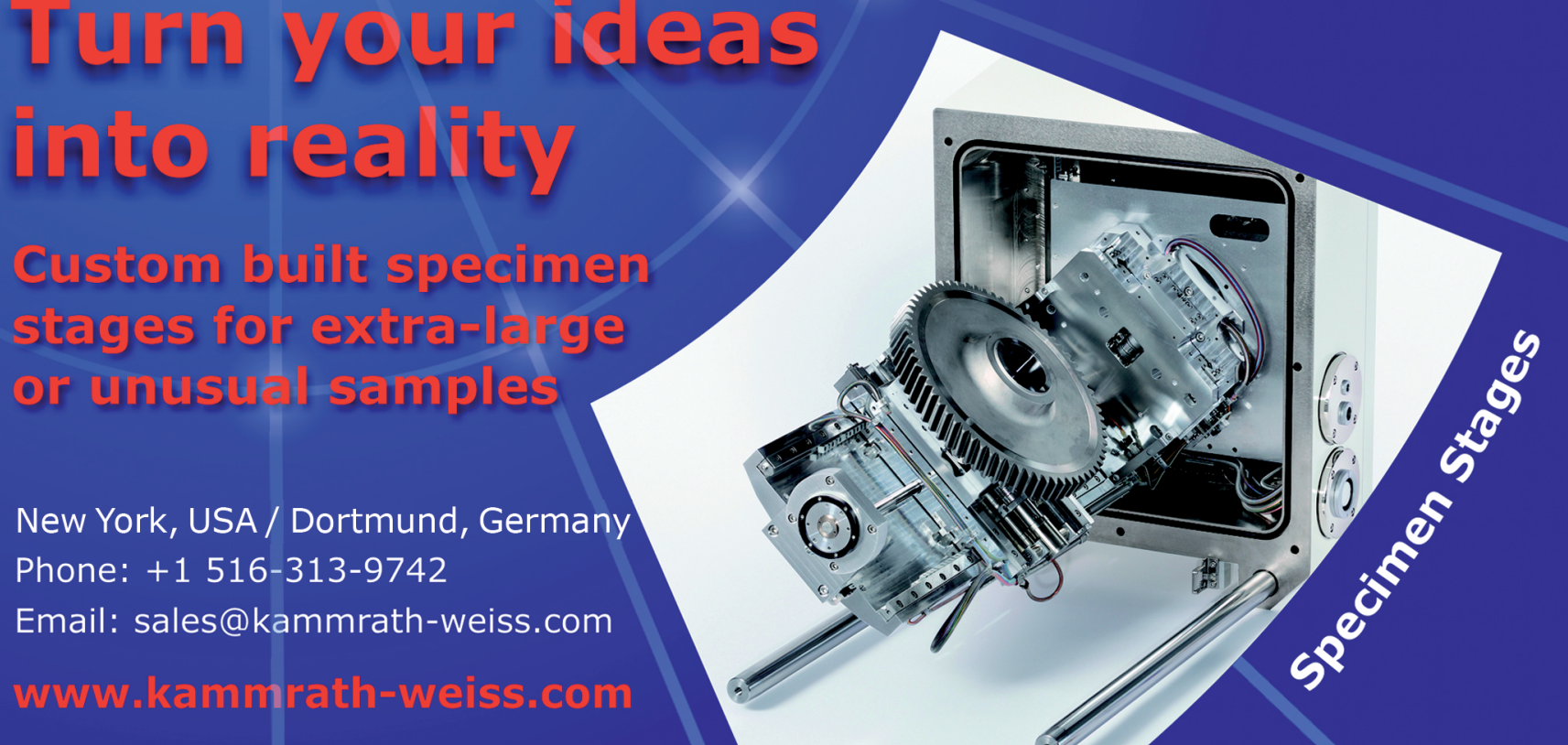

Buying pre-owned microscopes doesn't need to be risky. For more than 15 years, TSS has supported customers with a large inventory of meticulously remanufactured electron microscopes, plus accessories and spare parts. And our experienced team of engineers works with you to assure your happiness with your "new" microscope. Just like the OEM, we offer full support after the sale.

\section{Reunite with a Former Favorite that Still has Purpose}

Browse our inventory online or contact us if you need parts or service support for your legacy microscopes, even if you didn't purchase from us. We have engineers and customer representatives around the world, with our newest service and support center now open in Taiwan.
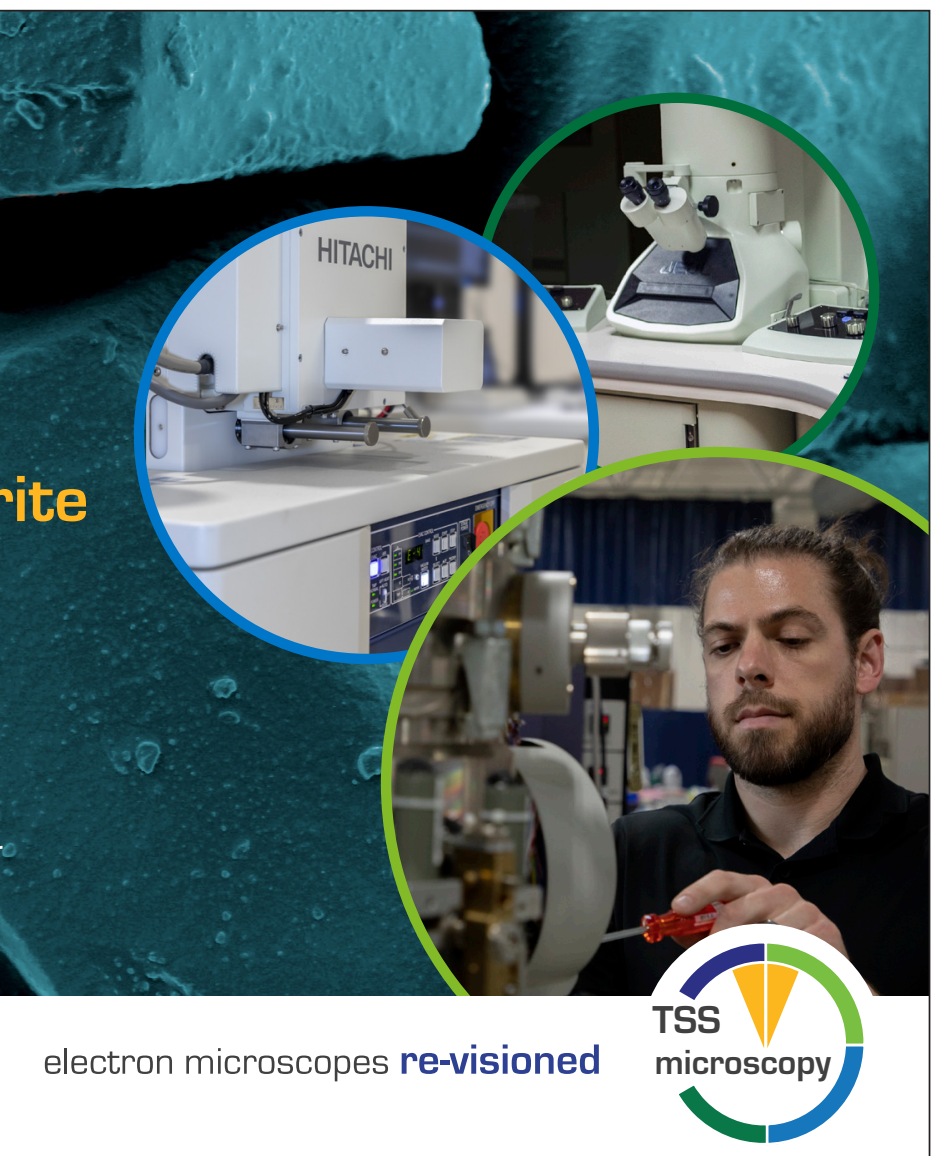